\title{
A Multiband MAC Protocol for Impulse-based UWB Ad Hoc Networks
}

\author{
Ioannis Broustis $^{\dagger}$, Srikanth Krishnamurthy ${ }^{\dagger}$, Michalis Faloutsos ${ }^{\dagger}$, Mart Molle ${ }^{\dagger}$ and Jeffrey Foerster * \\ $\dagger$ Department of Computer Science and Engineering, University of California, Riverside, Riverside, CA \\ * Intel Research, Hillsboro, OR \\ \{broustis,krish,michalis,mart\}@cs.ucr.edu,jeffrey.r.foerster@intel.com
}

\begin{abstract}
Ultra Wide Band (UWB) technology offers a promising high capacity solution for wireless networks with short-range links. However, MAC and higher-layer ad hoc network protocols that exploit the UWB technology are yet to mature. In this paper, we propose a MAC protocol for use in multi-hop wireless networks that deploy an underlying UWB based physical layer. We adopt a multi-band approach wherein we divide the available UWB bandwidth into multiple simultaneously usable bands. The motivation comes from the following observation: in the absence of a sophisticated equalizer, the size of a slot for transmitting a UWB pulse is typically dictated by the delay spread of the channel. Therefore, using a wider frequency band to shorten the transmission time for each pulse may not increase the data rate in proportion to the available bandwidth. Thus, we consider a multi-band approach to better utilize the available spectrum, where each transmitter sends longer pulses in one of many narrower frequency bands. Unlike previous single hopped schemes that rely on timehopping, our approach allows data transmissions to be contiguous, and thus, highly efficient. The approach also ensures that data communications are practically interference free and are only subject to thermal noise effects. To ensure that our proposed approach is tightly knit with the underlying physical layer, we discuss physical-layer dependencies and the conformance of our approach to FCC-imposed emission limits. We evaluate our approach via extensive simulations. Our simulation results demonstrate the significant advantages of our approach over single-band solutions: the throughput increases significantly, and the number of collisions decreases considerably.
\end{abstract}

Index Terms - Ultra Wide Band (UWB), Short-Range Communications, Medium Access Control, Ad Hoc Networks.

\section{INTRODUCTION}

Ultra Wide Band (UWB) is a novel wireless short-range technology, which has been the focus of a lot of interest in recent times [6], [10], [13], [14], [16], [17], [23]. Our objective in this effort is to design a MAC protocol that fully utilizes the capabilities of UWB communications. While physical layer technologies on UWB communications have been developed to some extent [14], MAC and

This work is supported in part by the NSF CAREER Grant No. 0237920 and the NSF NRT grant No. 0335302. higher layer technologies that enable the use of UWB in ad hoc networks are yet to mature [6]. The unique properties of UWB pose challenges to the design of a MAC protocol and requires the MAC layer to be synergetic with the underlying physical layer. We present three of these practical challenges, which motivate our multiband approach.

The first motivating observation is an artifact of the wireless channel effects on UWB transmissions. With impulse-based UWB, pulses are subject to multipath delay spread due to which, multiple time-shifted copies of each transmitted pulse appear at the receiver. This delay spread causes inter-symbol interference (ISI), wherein the delayed copies of one pulse interfere with subsequent pulses [3]. In indoor settings the magnitude of this delay spread is of the order of tens of nanoseconds. One approach to deal with ISI is the use of sophisticated equalization. However, this adds considerable hardware complexity to the transceivers and increases the synchronization overhead. In fact, UWB communications already require a long acquisition time for nodes to be synchronized prior to communications [18], which becomes longer due to the training sequence overheads required with equalizers ${ }^{1}$. Another approach to reduce ISI is to ensure that the spacing between the received pulses is larger than the delay spread; thus, the delayed copies of one pulse will not interfere with the next pulse ${ }^{2}$. With this approach, as opposed to the width of a pulse, the inter-pulse spacing constrains the throughput of the channel. Thus, in this case, $a$ band of smaller bandwidth, with an elongated pulse duration, can yield a throughput comparable to that of a wider band with a much narrower pulse duration. Hence, we note that we can partition the UWB spectrum into multi-

\footnotetext{
${ }^{1}$ We wish to point out here that the Multiband OFDM Alliance supports an OFDM (Orthogonal Frequency Division Multiplexing) based specification [5] for UWB; the motivation for dividing the available spectrum into multiple bands is to overcome the need for complex equalization. OFDM however, first requires complex signal processing in terms of complex inverse fourier transform computations. Second, a MAC protocol for use with OFDM for UWB-based ad hoc networks has yet to emerge.

${ }^{2}$ For a given average power constraint, the peak power constraint also imposes restrictions on the pulse repetition frequency (PRF) as we will discuss later.
} 
ple comparatively narrow frequency bands that are mutually orthogonal and can be used simultaneously, and thus, use the available spectrum more efficiently.

The second motivating observation stems from the absence of carrier sensing capabilities in UWB. With impulse-based UWB, data is transmitted in the form of pulses $^{3}$ and there is no contiguous carrier, although these pulses are possibly modulated by means of a high frequency signal (referred to as the pseudo-carrier). Thus, the commonly used protocols that rely on carrier sensing are not necessarily applicable with UWB. In addition, the very limited number of UWB based MAC protocols that have been proposed previously are based on arbitration via time-hopping on a single channel. But, time-hopped sequences with a short spacing between the time-hops can lead to collisions, while long durations between time-hops can lead to excessive delays and low efficiency. Thus, the second key objective of our design is to reduce collisions to the extent possible, without resorting to long time-hopping sequences.

The third motivation for our multiband approach is the associated flexibility in spectrum use and the interoperability with other networks. If a portion of the UWB bandwidth is being used by other coexisting services, the corresponding band can be avoided with a multiband approach. Thus, UWB communications can coexist with other networks (such as IEEE 802.11a based networks), a definite requirement in urban, disaster recovery and military settings. For example, in the presence of an IEEE 802.11a network, the multiband system can avoid using the bands centered at $5.35 \mathrm{GHz}$ or $5.85 \mathrm{GHz}$. We also wish to point out that the multi-band transceiver circuit remains simple [20], i.e., the cost, power and integration concerns are similar to those in a single-band system.

Thus motivated, we propose and develop a novel multiband MAC protocol for use with UWB based ad hoc networks. To the best of our knowledge, this is the first multiband MAC protocol that is synergetic with UWB communications and is designed for use in ad hoc networks. The key concept of our design is the use of different bands for control and data transmission (the separation is not pure as we will see later). Simply put, two nodes first use a control channel to facilitate a rendezvous in another band for a data exchange. The first advantage of the approach is that, since all the nodes share a common unreserved channel only for short control messages, the contention on the shared channel is limited. Second, once a pair of nodes agrees to communicate on a data band, the communication

\footnotetext{
${ }^{3}$ Recent developments with OFDM and Multi-carrier CDMA use carrier based methods; the trade-offs between the use of impulse-based UWB and OFDM based UWB are discussed in [14].
}

can be continuous (no need for the use of time hopping sequences), and thus, it is highly efficient. This efficiency is also enhanced by the fact that, once a communication is established in a data band, our protocol practically eliminates the possibility of collisions of transmissions of large data packets. The throughput of our scheme is significantly higher compared to a single-band approach that combats delay spread by increasing the spacing between pulse transmissions. In addition, the number of pulse level collisions also drops dramatically.

Finally, we wish to point out that UWB communications are constrained by emission limits imposed by the Federal Communications Commission (FCC) [1]. In particular, FCC requires that the effective isotropic radiated power (EIRP) be no higher than $-41.25 \mathrm{dbm} / \mathrm{Mhz}$. Our design conforms to FCC requirements both in terms of the average and peak emission power levels.

The remainder of this paper is organized as follows. In section II, we provide the relevant background on UWB communications, discuss the physical layer dependencies and demonstrate the conformance of our design to FCC imposed regulations. In section III, we provide a detailed description of our protocol. In section IV, we present our simulation framework, results and deliberate on the observations. Related work on the design of MAC protocols for use with UWB is discussed in section V. Finally, section VI concludes the paper.

\section{Physical Layer Dependencies}

In this section, we discuss the UWB physical layer and highlight its impact on the design of our protocol. Detailed descriptions of some of the aspects of UWB communications can be found in [1], [13] and [14].

Facilitating Multi-band Impulse-based UWB Communications: UWB communications, as per the specifications of the FCC, use the spectrum from $3.1 \mathrm{GHz}$ to 10.6 GHz [1]. FCC defines UWB communications as those that use signals that span at least $500 \mathrm{MHz}$ of absolute bandwidth or those that occupy a fractional bandwidth $W / f_{c} \geq 20 \%$, where $W$ is the transmission bandwidth and $f_{c}$ is the frequency at the center of the band [13]. UWB systems have traditionally achieved these high bandwidths by using pulses that are of very small or narrow time duration; we refer to these as impulse-based UWB systems. A typical UWB pulse belongs to the family of Gaussian shaped doublets [13], [14]; these shapes are generally used since they can be easily generated by hardware. The shape approximates a Gaussian function curve. A Gaussian pulse may be represented by

$$
s(t)=K e^{(t / \tau)^{2}},
$$


where, $K$ is a constant that reflects the amplitude of the pulse and thus, the peak transmission power ${ }^{4}$ [14].

Multi-band modulation facilitates the division of the 7.5 $\mathrm{GHz}$ of spectrum made available by the FCC into multiple smaller frequency bands. The spectrum allocated to each band must meet the FCC specifications as mentioned earlier. With impulse-based UWB, the pulse shape is the primary characteristic that determines the distribution of energy in the frequency domain and therefore allows for the separation and thus, the simultaneous use of the bands. Depending on the spectrum of operation, the Gaussian pulse defined in Equation 1 is modulated by a set of carriers that belong to the particular band. This central frequency component is typically referred to as the pseudo-carrier. Note here that these high frequency modulating signals are simply used to shape the pulse and are not used to reflect symbol information as in traditional modulation methods wherein a carrier is modulated (such as frequency shift keying or FSK [2]). By properly shaping the pulse, one may ensure that most of the energy is concentrated near the center of the desired band thereby reducing the possibility of inter-band interference. We wish to also point out that the central frequency components of the different bands must be separated sufficiently in the frequency domain to avoid inter-band interference effects. A detailed discussion of pulse shaping can be found in [14]. In our simulations, we use a simple Gaussian shaped pulse and assume that appropriate modulating signals can be employed (as shown in [14]) to facilitate the division of the bandwidth into multiple bands each of which is $500 \mathrm{MHz}$ in bandwidth. The pseudo-carrier of the highest band is $10.35 \mathrm{GHz}$ and that of the lowest band is $3.35 \mathrm{GHz}$. The pulse-width will depend on the wavelength of the pseudo-carriers being used. Since the wavelength of the pseudo-carrier in the multi-band case could be potentially larger (depending on the band) than that in a single band case (wherein the entire allocated spectrum is used), the pulse-width would be longer with multiband impulse-based UWB.

Encoding Information: Pulse Position Modulation: The modulation scheme that we use is a commonly deployed scheme called Pulse Position Modulation or PPM $[14]^{5}$. We also assume the use of a rate $1 / 3$ convolutional code [3] which in turn implies that the information in each bit is encoded into three symbols. Each pulse represents a

\footnotetext{
${ }^{4}$ We discuss power constraints later. Typically the value of $K$ is dictated by FCC regulations.

${ }^{5}$ We wish to point out here that multiband systems may be based on direct sequence CDMA modulation or OFDM. For this work, we use PPM modulation. While no CDMA or OFDM based MAC layer solutions have been completely developed for ad hoc networks, we discuss relevant related work in a later section.
}

symbol. The information in the symbol (whether the symbol represents a " 0 " or " 1 ") is determined by the position of the pulse within what we call a chip time $T_{c}$. If the pulse occupies the first part of the chip-time, it represents a symbol value of " 0 "; else, a symbol value of " 1 " is implied. We assume that a Viterbi decoder is deployed at the receiver [2] and this enables the soft-decision decoding of the received information.

Time Hopping: Time hopping has been used in previous approaches for sharing a single frequency band among multiple users [6], [7]. However, we use this approach only in the control band and not in the data bands as we explain later. In time hopping, a fixed number of chiptimes are aggregated to form a sequence frame. The duration of each sequence frame is $T_{f}$, and thus the number of chip-times per sequence frame is $T_{f} / T_{c}$. Each transmitter sends a pulse in only one of the chip-times in each sequence frame. The specific chip-time is determined by the node's time hopping sequence (THS), which is typically generated via a pseudo-random number (PN) code. The distribution of PN codes (for making a node's THS known to its neighbors) has been the topic of a few efforts [21], [22]. In our work, we assume that the PN code is a function of a node's identifier (possibly the MAC layer address). The generators of these PN code sequences are initialized at system set up. Nodes periodically use outof-band techniques to announce the state of their PN code generators ${ }^{6}$. The technique is similar to the proposal in [21].

Time hopping sequences may be either sender-based or receiver-based. In receiver-based time hopping, a receiver expects to receive a pulse only in one of the chip-times in a sequence frame. In the sender-based case, the transmitter sends pulses based on its own THS. The senderbased strategy is robust; however the receiver has to be synchronized with all of its potential transmitters at the same time. The receiver-based approach is much simpler to implement; however one could encounter collisions between the pulses from different transmitters, directed towards the same receiver. It is possible for protocols to use both approaches, as in [6].

The average spacing between successive transmissions as per the THS will have an effect on the achieved performance. With shorter spacing between the time-hops, the pulses could be sent at a faster rate ${ }^{7}$; however, there is a higher possibility of collisions. With longer spacing, the possibility of collisions is reduced; however, large de-

\footnotetext{
${ }^{6}$ These announcements are made in special frames that we refer to as Availability frames. We discuss this in a later section that describes our protocol.

${ }^{7}$ The FCC regulations impose a limit on the pulse repetition frequency as will be discussed later.
} 
lays could be incurred. With our scheme, as mentioned earlier, time hopping is only used for the transfer of short control messages; since these messages are infrequent and fairly short in duration (low load), the probability of experiencing collisions remains low even with a relatively short spacing between the time-hops.

Channel Impairments and Effects: We next discuss the effects of the wireless channel on UWB communications and the associated impact on our MAC protocol design. A signal typically experiences three types of channel impairments: pathloss, shadowing and multipath effects. The pathloss is given by the Frii's law [13]:

$$
\alpha=\left(\frac{c}{4 \cdot \pi \cdot d_{i j} \cdot f_{c}}\right)^{2}
$$

where $c$ is the speed of light, $f_{c}$ is the central frequency of the band and $d_{i j}$ is the distance between the transmitter and the receiver. Note that the above equation depicts the observed effects on average, and does not imply that each transmitted signal experiences the same level of attenuation. Furthermore, at a given distance $d_{i j}$, higher frequencies will experience higher levels of attenuation than lower frequencies. We ignore shadowing effects since we assume that transmissions are typically over short distances $(\approx 10$ meters $)$ and therefore do not experience shadow fading [19].

Due to the multipath nature of the wireless channel, UWB transmissions (high data rates) will experience multi-path delay spread. A transmitted UWB pulse, radiated using an isotropic antenna, will take multiple paths (as a consequence of reflections from various objects) to arrive at the receiver. In other words, the receiver will receive multiple copies of the same signal, each of which may have a different amplitude, phase and delay. Beyond a certain delay threshold (an inherent characteristic of the channel being considered), the signal amplitudes may be considered negligible. This threshold is referred to as the delay spread of the channel. For indoor environments, measurements have shown that the delay spread is of the order of tens of nanoseconds [19]. If the time-spacing between the UWB pulses is smaller than the the delay spread of the channel, copies of the a transmitted symbol interfere with the subsequent symbols. This is called inter-symbol interference or ISI for short. Equalizers are typically used to combat ISI [2]. The higher the level of the ISI, the higher the complexity and sophistication of the required equalizer. Equalizers also require the transmission of a training sequence prior to information communication. This can be expensive in terms of the overhead consumed. With UWB transmissions, a preamble is needed to allow for the sender and receiver to synchronize prior to communications. By acquisition, we mean that the receiver learns how to recognize the presence of a pulse train in the presence of thermal or other noise factors. The aforementioned acquisition preamble is considered expensive in terms of overhead [18]. The deployment of a sophisticated equalizer will further increase the overhead costs incurred with UWB.

Another strategy for combatting ISI would be to use direct sequence CDMA in conjunction with a Rake receiver. However, the long codes with CDMA could still incur capacity penalties. Furthermore, with CDMA, the sender and receiver require code synchronization in addition to the acquisition and this would incur a further cost in terms of overhead.

The alternative that we explore in this work is to separate the pulses by at least the delay spread of the channel. Thus, the time-spacing between the pulses is chosen to be at least $30 \mathrm{~ns}^{8}$ (delay spreads in indoor environments [19]). We recognize that by doing so, the pulse width could be increased to some extent since this is unlikely to interfere with future symbols. Increasing the pulse width allows for the use of lower pseudo-carrier frequencies and thus, facilitates the use of multiple frequency bands as discussed earlier. Thus, this facilitates a multi-band approach.

Conformance with FCC regulations: The FCC regulations limit the effective isotropic radiated power (EIRP) to $-41.25 \mathrm{dBm} / \mathrm{MHz}$ (Part 15 of the regulation) [1] [13]; the power used, on average, per bit cannot exceed this imposed limit. Let us denote the transmit power by $P_{T}$ $\mathrm{dBm} / \mathrm{MHz}$, the received SNR at a distance $d$ by $S N R_{d}$ $\mathrm{dB} / \mathrm{MHz}$ and the central frequency in the band used by $f_{c}$. Let the power spectral density of the thermal noise be $N_{o} \mathrm{dBm} / \mathrm{MHz}$. Then, the signal to noise ratio is given below[13]:

$$
S N R_{R}=P_{T}-N_{o}-20 \log \left(\frac{4 \pi f_{c}}{c}\right)-20 \log d .
$$

If $P_{T}$ is $-41.25 \mathrm{dBm} / \mathrm{MHz}$ and if the received power i.e., $P_{R}$ is to be $3 \mathrm{dBm}$ higher ${ }^{9}$ than the noise margin wherein $N_{o}=114 \mathrm{dBm} / \mathrm{MHz}$ [13] one can compute the maximum range of transmission for the given value of $f_{c}$. When computing this, no coding is assumed ${ }^{10}$. Note here that, the higher the value of $f_{c}$, the lower the maximum range. For the highest frequency band where $f_{c}=10.35 \mathrm{GHz}$,

\footnotetext{
${ }^{8}$ Note that this translates to having a chip-time of $60 \mathrm{~ns}$.

${ }^{9} \mathrm{SNR}=3 \mathrm{~dB}$.

${ }^{10}$ Note that with additional coding (rate $1 / 3$ convolutional code), extremely low bit error rates (of the order of $10^{-8}$ ) can be achieved with these parameter settings.
} 
this translates to a range of around 7 meters. We set the range to 7 meters for all of the other frequency bands and compute the average transmission power for those bands. The appropriate powers are then used so that the range is identical, irrespective of the band being used. Clearly, the powers used will be lower than the FCC imposed limit. As an example, for the lowest spectral band, the value of $f_{c}=3.35 \mathrm{MHz}$ and for a range of 7 meters, the average transmission power is $-51.158 \mathrm{dBm} / \mathrm{MHz}$ which is lower than the FCC imposed limit. Note here that the value of the constant factor $K$ in Equation 1 (previously discussed) can be then determined based on the power used for transmission. To summarize, with the settings as above, we conform to the FCC imposed restrictions on the EIRP.

In addition to the imposed restriction on EIRP, the FCC also imposes a limit on the peak power that can be used for UWB transmissions. As specified in [1], if the average power limit is met and the frequency of pulse transmissions is higher than $1 \mathrm{MHz}$, the peak power limitation is also met. With our scheme, since the maximum distance between the pulses is $60 \mathrm{~ns}$ (the chip-time), the frequency is $16.67 \mathrm{MHz}$. This implies that our scheme inherently conforms to the peak power constraint.

Coding and Higher Layer Abstractions: As mentioned earlier, we assume that a rate $1 / 3$ convolutional code is used for encoding data. This code can help efficiently recover from errors if the only impairment is thermal noise. In our control band, we use a THS for arbitration as we will discuss later. This can in turn lead to collisions among transmissions. In order to provide a further level of robustness on this band, we employ a repetition code of 2. In other words, we repeat twice the output of the convolutional encoder, in order to provide robustness to collision effects.

In our simulations, we assume the presence of the convolutional encoder and decoder and do not implement them. Instead, we use the bit error rate of $10^{-7}$ and discard bits at this rate. This error rate almost never results in any lost packets due to bit errors.

Time Synchronization: Our approach requires the division of time into frames, which implies that communicating nodes must be synchronized in time. We assume that synchronization is achieved by the use of previously proposed methods for this purpose [25], [26].

\section{The Multi-BAND MAC PRotocol}

In this section we present our multi-band MAC protocol. The key idea is to have a communicating pair of nodes exchange data over a private band as opposed to a single common band, as in single band systems. In the private band, we would not have to use time hopping and thus we

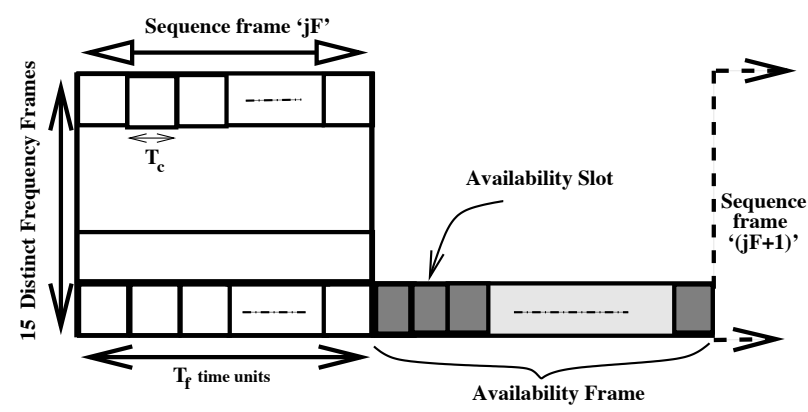

Fig. 1. The frame structure with our protocol

avoid the disadvantages of using time hopping sequences (THS) as discussed earlier. We first give a brief overview of the basic concepts and the operation of the protocol.

The Multiple Bands: We divide the available frequency bandwidth into $B$ bands. $B-1$ of these bands are used for data transmissions and are referred to as data bands. The remaining band is used for request control packets only; we call it the Request Band or Req-Band; the first band is assigned to be the Req-band. As discussed earlier, if each band is of bandwidth $500 \mathrm{MHz}, B=15$. The protocol is designed based on the physical separation of the available UWB bandwidth of $7.5 \mathrm{GHz}$ into multiple bands (as discussed in the previous section) each of which spans $500 \mathrm{MHz}$ of the spectrum ${ }^{11}$.

The Frame Structure: Across all the bands, the time is broken into superframes which are separated by smaller availability frames. All data and control communication takes place during superframes. The availability frame is used to indicate the status of the multiple bands, namely whether the bands will be busy or not in the next superframe as we will explain later. The purpose of the availability frames is to alleviate the possibility of collisions of data transmissions in the superframes. Note that each superframe consists of $F$ sequence frames, each of which in turn consists of $T_{f} / T_{c}$ chip-times as described in section II. In Figure 1, we depict the frame structure; the availability frame is sandwiched between the last sequence frame of the $j^{\text {th }}$ superframe and the first sequence frame of the $(j+1)^{s t}$ superframe.

Protocol Operations in a Nutshell: We first provide a high level overview of our protocol. The protocol implementation at each node can be represented by a finite state machine as shown in Figure 2. Initially, a node is in the IDLE state. When data needs to be sent, it enters the REQUEST state as shown. In this state, it attempts to initiate a request to the appropriate receiver. Towards this, it transmits the request as per the receive THS of the receiver in the Req-band. If this request were to succeed, the node enters the TALK state, switches to a data band

\footnotetext{
${ }^{11}$ Note that FCC specifications require that each UWB band is at least $500 \mathrm{MHz}$ in bandwidth.
} 
(the rules for choosing a band will be discussed later) and attempts to establish a connection with the receiver. If the request were to fail, it enters the BACK-OFF state and tries again at a later time. After successfully establishing a communication, the node sends data. In addition, it periodically announces (by transitioning to the DECLARE state), by means of the availability frame, that the specific data band being used is occupied. This precludes other nodes from claiming the particular band and causing collisions. Upon the completion of the data transfer, the node returns to the IDLE state.

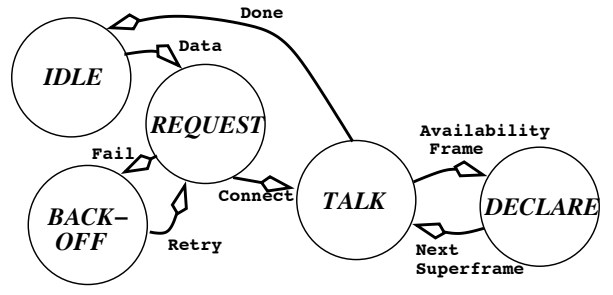

Fig. 2. Depiction of Protocol Operations

Detailed Protocol Descriptions: Next, we discuss the protocol in greater detail and in particular, the nuances of protocol operations in each of the aforementioned states.

Request Initiation: Upon having data (either its own or data that it has to forward) to send to a neighbor, a node will first have to send a request to the receiver. Our design mandates that transmissions are to be initiated at the beginning of each superframe, i.e., right after the availability frame. The availability frame (as we will discuss later) reflects the occupancy of each of the data bands. By the above design mandate, we ensure that nodes have up-todate information on which of the data bands are occupied prior to initiating new transmissions. This would prevent these new transmissions from colliding with previously initiated data transfers that might be in progress. Thus, if a node (whose queue was empty at the end of a particular availability frame) generates packets for transmission in the middle of the following superframe, it is precluded from initiating a transmission before the end of the upcoming availability frame. At these allowed times, in order to initiate a request, the sender sends a $R E Q$ packet in the Req-Band as per the THS of the receiver. The REQ packet identifies the particular band that the sender has chosen for the data exchange. After transmitting the REQ packet, the sender switches to the indicated data band and awaits a response from the receiver. Note that the above operations occur in the REQUEST state discussed earlier.

Acknowledgment of the request: If the REQ packet is correctly received, the receiver will switch to the specific data band indicated in the REQ packet, and will send a RACK (Request Acknowledgment) packet to originating sender. If the RACK packet is successfully received by the sender, it completes a successful handshake and the sender can then begin the data transfer.

Transfer of Data: The reception of the RACK asserts that the band is almost surely free for exclusive use for data transfer. In the chosen band, nodes (now in the TALK state) transmit data in consecutive chip-times instead of using time hopping. As discussed in the previous section, the spacing between the pulses is at most $60 \mathrm{~ns}$ and we ensure that the FCC emission regulations are met. Upon the successful reception of a complete data packet, the receiver sends a DACK (Data Acknowledgment) packet to the sender. Even if collisions are completely eliminated, it is possible that other noise factors (thermal noise) can corrupt the data packet. If the receiver is unable to correctly decode the packet, it does not issue a DACK back to the sender. The sender would then reattempt to transmit the data packet up to a fixed number of times, after which the packet is dropped.

The Availability Frame and the DECLARE state: As mentioned earlier, superframes are interspersed with the so called availability frames. During the much smaller availability frame, data communications stop temporarily so that nodes currently occupying a data band can signal their intention to continue using it during the next superframe. This signaling takes place in the Req-Band (we could have chosen any band, since availability frames are exclusively used for signaling availability and no data transfers occur during these frames). The availability frame is divided into time intervals that are different in size from those in the sequence frames. The number of these intervals corresponds to the number of data bands. We call these intervals availability slots. Communicating nodes "saturate" the availability slot that corresponds to the data band that they intend using in the next superframe. As an example, if a communicating pair is using data band $j$ where $2 \leq j \leq B$, the pair would transmit in the $j^{\text {th }}$ slot of the availability frame. The sender saturates the first half of the availability slot and the receiver the second half. This is done to ensure that the neighbors of both the sender and the receiver are made aware that the corresponding band is occupied. Nodes in search of an available band listen to the availability frame and select an unused band for their upcoming data transfers. Note that, due to the consecutive transmission of pulses during the availability frame, nodes are able to detect (or sense) the pulses. The size of each availability slot is chosen so as to accommodate an adequate number of pulses to facilitate acquisition and to combat noise effects. The availability frame corresponds to the DECLARE state discussed earlier.

Choosing a band for communication: Initially each 
sender selects a band randomly from the set of free bands, as indicated by the availability frame. The following mechanisms are incorporated to further reduce the possibility of collisions due to multiple new senders choosing the same band.

1) Persistent Band Selection: Nodes keep a history of bands that they successfully used in the past. The random choice process is biased with time such that the nodes would prefer to re-use these previous successfully used bands. In the long run, this can further reduce the possibility of two (or more) senders selecting the same band. This can be particularly helpful when traffic is bursty and the same sender nodes are active for repetitive interspersed busy and idle periods. Note that in most practical networks, traffic and communication patterns are indeed bursty [24].

2) Availability Eavesdropping: Nodes can determine the bands that are being used even when they do not have packets to send, by means of of the availability frame. Thus, they can keep track of bands that are consistently occupied (as per persistent band selection). A band that is often busy is more likely to be used in the future. Thus, new senders can avoid the use of these bands.

In fact, the two mechanisms discussed above, enable a self-organizing behavior, where groups of nodes that have disjoint periods of activity end up having the same preferred bands. A trivial example is the case of two nodes sharing two bands: persistence and eavesdropping can lead to each node using a band without conflicts. This can be generalized to a case with any number of nodes and bands.

Failure of the Request Process and the BACK-OFF state: There are three cases where the receiver does not reply successfully to the sender with a RACK: (a) there were more than one REQs that collided, (b) the receiver is busy, and (c) two or more pairs of communicating nodes attempt to use the same data band. To elaborate on case (a), if two nodes (or more) transmit their REQs to a common receiver at the same time, a collision will occur. In this case, the two senders after the REQ transmission will switch to their own selected data bands and will wait for a response from the common receiver. As a result of the collision of the REQ packets, they do not receive a response. The sender nodes wait for a specified time interval in their selected bands, and at the end of this period they conclude that a collision has occurred. They will then initiate backoff timers and at the end of their back-offs, reattempt to initiate the request.
We employ a simple additive back-off scheme ${ }^{12}$ for retransmission attempts after a failure. Upon experiencing a collision, a sender chooses, with a uniform probability, one of the $M$ subsequent superframes to reattempt its request. The number $M$ is given by $M=N+x L$, where $x$ is the number of consecutive failures and $N$ and $L$ are system parameters that define the aggressiveness of the back off policy. We impose a maximum limit on the number of retransmission attempts $x$, after which the packet is dropped.

To elaborate on case (b), if the receiver is busy in another data band either sending or receiving data, it does not receive the REQ packet. The sender will, as in the previous case, transmit the REQ packet and await the RACK packet in the data band of its choice. Clearly, in this case, no RACK packet is forthcoming. The sender cannot distinguish this case from case (a) in which a collision occurs. Therefore, it enters the BACK-OFF state as discussed earlier and reattempts a request at a later time.

In case (c), if two or more pairs of nodes select the same band, their transmissions may collide in that data band. The problem is exacerbated, when the number of sender nodes is much larger than the number of bands. This problem is alleviated to a large extent by our policy of initiating new transmissions only at the beginning of a superframe. Thus, when two pairs of nodes choose the same band their RACK packets collide. The nodes would infer that a collision has occurred and will retract to reattempt a reservation. Note that the collision is quickly and efficiently detected.

Enabling receptions while in back-off: While a sender node's back-off counter is counting down, the node switches to the Req-Band. In this band, it resumes receptions as per its THS while its backoff counter ticks down. If the node successfully receives a preamble for a REQ packet (from any of its neighbors), the node temporarily freezes its backoff timer and switches to the band specified in the REQ packet, to attempt a reception from the originator of the REQ packet. Upon the completion of the reception, or upon the detection of a collision, the node under discussion would switch back to the Req-Band and resume the countdown of its back-off timer. Without this, nodes that are attempting to contact the sender under discussion would be forced to back-off, while the node is counting down. This would degrade the efficiency of the system, since a node can be blocked waiting to send to another blocked node forming a chain or even a cycle.

Finally, while awaiting RACK, Data or DACK packets, a node will wait only up to preset time limits (system pa-

\footnotetext{
${ }^{12}$ Our simulations suggest that this simple scheme is very effective in resolving collisions.
} 
rameters). If the expected packet is not received within this time limit, the node assumes that the communicaton has failed. The sender would then attempt to resend a request after an appropriately chosen back-off time.

Multi-hop Communications: Coping with Hidden Terminals: The hidden terminal problem is already alleviated to a great extent since the transmitter and the receiver both send messages in the availabilty band to indicate the occupancy of the band on which they currently communicate. This ensures, to a large extent that neither the neighbors of the sender nor those of the receiver claim the same band. However, note that after transmitting their REQ packets, the nodes rendezvous in the chosen data band. Since the REQ packets are sent according to THSs, the transgression of communicating pairs onto data bands is not synchronized. Thus, it may happen that two pairs choose the same bands but move to that band for the rendezvous at different times. Now, if, after such a rendezvous between a given sender and a receiver, a neighbor of the receiver, hidden from the sender, switches to the same band and initiates a new message transfer, a collision would occur at the receiver. In order to avoid such effects we require that: (a) when a pair of nodes switches to a new band for data transfer, they wait for a duration of $T_{n}$ nanoseconds (during which they listen to other possible communications on the band) prior to completing their handshake and beginning the data transfer, and (b) receivers send short occupancy indication messages in the band on which they are on with a periodicity of $T_{n}$ nanoseconds. This will further reduce the possibility of collisions due to hidden terminals. In fact, our simulations suggest that the two schemes together practically eliminate collisions.

\section{Simulation Results}

We present the evaluation of our idea through simulations using a $\mathrm{C}++$ simulator that we have developed by extending a previous simulation effort [10]. Our focus is on the performance at the MAC layer. Thus, we assume that data is injected at the MAC layer and the transmissions of a node are intended for a neighbor. However, we wish to clarify that nodes are distributed over a region of interest for multi-hop operations; thus, MAC layer effects such as the presence of hidden terminals are accounted for, in our simulations. In our simulations, we use assumptions and conventions that are widely used in UWB studies and try to incorporate as many realistic details [6], [10], as possible. Some of our simulation assumptions were alluded to in Section II.

Comparisons: We compare our scheme with an a single-band approach in order to demonstrate the benefits of our multi-band scheme. All data and control packets use the entire $7.5 \mathrm{GHz}$ bandwidth in the single-band approach, whereas up to $B-1$ simultaneous users can transmit data packets on different bands during the same superframe in our multi-band scheme. Thus, to avoid giving our scheme an unfair advantage, we magically eliminate the effects of pulse collisions on the reception of data packets in the single-band approach: communicating nodes switch to a separate predetermined THS to exchange data packets ${ }^{13}$. Note that this assumption now shifts the unfair advantage to the single band case, since many more than $B-1$ simultaneous data transfers could be supported if the requests get through ${ }^{14}$. One can envision this to be akin to using a perfect equalizer, which is calibrated during the reception of a request packet, to eliminate the ISI during the reception of the following data packet. Note however, that with both the single and the multi-band approaches, pulse collisions may occur during the initial handshake wherein a request is transmitted (we refer to this as request transmissions in Section III) as per the receiver's THS.

Simulator Implementation Details: In our implementation, the physical layer consists of a number, $m$, of sets of virtual links as shown in figure 3 . This number is equal to the number of bands; each set of links has a separate buffer and connects a node with its neighbors ${ }^{15}$. As a result, a node has $m$ links with a neighbor node, each representing a different band. The MAC layer of the transmitter delivers the packet to the appropriate link of the appropriate band. The physical layer component converts the bits to pulses, which will be transmitted through this link. The channel characteristics, discussed earlier in Section II are applied and distort the transmission. The receiver picks each pulse, decodes a set of pulses that form a bit if possible, and stores the bit in a buffer. A bit may be discarded either due to a collision (elaborated below) or due to its being corrupted by thermal noise as discussed in Section II. When a set of bits that form a packet have been received correctly, the packet is re-constructed and delivered to the receiver's MAC layer. The arrival of two or more pulses, simultaneously from different links of the same band, denotes a collision.

\section{Simulation Scenarios:}

Network Layout: The nodes are mobile and form an

${ }^{13}$ A similar single-band scheme is described in [6]

${ }^{14}$ However, we wish to point out there may be other single band approaches that simply have pulse boundaries that commensurate with the actual duration of the pulse as opposed to the delay spread. This may lead to a higher number of pulse collisions; however, at the same time, higher levels of redundancy may be employed given that more pulse transmissions are potentially possible. A systematic evaluation to determine the best possible single band approach is beyond the scope of this work.

${ }^{15}$ Note that two neighbors may have more than just their common neighbors. 


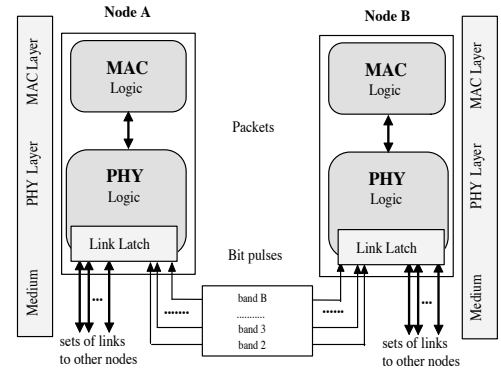

Fig. 3. Simulation Implementation Platform

ad hoc network. We vary the number of nodes from 6 to 30. We restrict the nodes in a $30 m \times 30 m$ square region and this is seen to maintain an average node degree larger than 3. As mentioned in section II, the maximum range of a transmitter is considered to be 7 meters. The total number of bands in the multi-band system is 15 , as mentioned earlier. A transmitter always selects a receiver randomly from within its transmission range.

Frame Structures: Every sequence frame consists of six $T_{c}$ frames (chip-times). The duration of the superframe is set to 11200 chip-times, which is approximately equivalent to a successful packet exchange including the control overhead. We divide each availability frame into $B-1=14$ availability slots, each of which is $33 T_{c}$ units of time in duration. This duration is sufficient for neighbor nodes to detect the pulses and correctly infer that the corresponding data band is occupied.

Traffic Characteristics: We use both CBR (Constant Bit Rate) and bursty Poisson traffic in our simulation experiments. In the experiments shown, the packets are of size 250 bytes and with CBR, are transmitted once every $40 \mathrm{msec}$, unless otherwise stated. Each control packet is 120 bits long, in accordance with the control packets used with other wireless protocols, such as with the IEEE 802.11 MAC protocol [11]. Even though the per-source CBR rate is low, with a multiplicity of sources, the load on the network fits in with the use of UWB.

Pulse collisions and bit errors: We assume the presence of a rate $1 / 3$ convolutional encoder for the data bands and accordingly, three pulses represent a single bit. In the control band or in the case of the single band system, in order to provide robustness to collisions, we use a repetition code of 2 , i.e., each bit (comprising of three pulses) is repeated twice. Thus, in these bands, six pulses form a bit. A pulse collision occurs when two or more pulses arrive during the same $T_{c}$ period, in the same band. A bit is received in error, when any of the pulses that make up the bit collide or if it is corrupted due to thermal noise.

Mobility Model: We assume that nodes move as per a Brownian motion mobility model. Each node chooses a

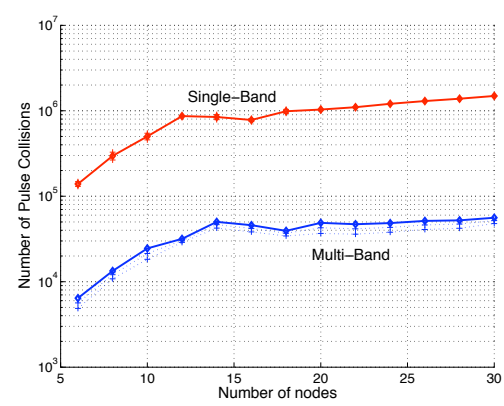

Fig. 4. Number of pulse collisions. Logarithmic scale is used

new position that differs from its current position by at most $10 \mathrm{~cm}$ in a randomly chosen direction, once every 6 milliseconds.

Back-off Policy: With our backoff algorithm (discussed in Section III) for packet retries, we set the initial back-off to a randomly chosen value between 0 and 5 superframes. After each retry, the maximum value increases by 2 , until it reaches a maximum of 15 . We have varied these values and the results obtained demonstrate behavioral traits that are similar to those considered in our sample set presented here. The packet is discarded if, after 15 attempts, a node is unable to deliver it to its intended neighbor.

Providing for consecutive packet transmissions: With any given reservation, we allow a transmitter to send two consecutive packets to its receiver. This would in some sense amortize the preamble and request costs over a larger transmission. We restrict this number to two to prevent the dominance of a channel by a single communicating pair. The overall simulation time is 15 million chip-times $T_{c}$.

Performance Metrics: We evaluate the performance of our scheme by measuring the number of pulse collisions, the bit error rate, the overall number of transmitted data packets during the simulation, the average packet delay and the utilization of a band. We define the metrics in detail when we discuss the results. In a few of the following graphs, we include $95 \%$ confidence intervals.

Results: Due to space constraints we only present a sample set of results. We first present results when CBR traffic is considered.

In figure 4, we plot the total number of pulse collisions for each approach as a function of the number of nodes in the network. We observe that our protocol decreases the number of pulse collisions by an order of magnitude as compared with the single-band approach. The reason is that, in our protocol, data packets are transmitted practically free of collisions, since they are exchanged on an exclusively reserved data band. In contrast, in the singleband case, packets suffer frequent collisions due to overlaps between nodes' THSs.

In figure 5, we plot the bit error rate averaged over the 


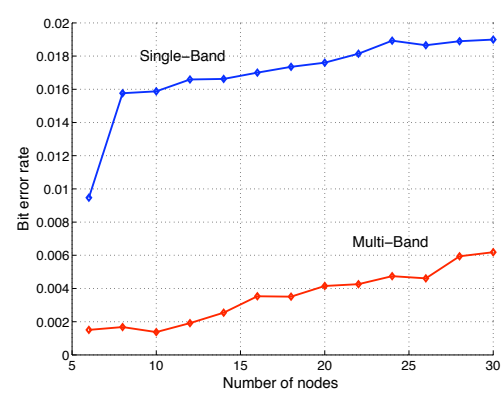

Fig. 5. Bit error rate in the network. The Multi-band scheme outperforms

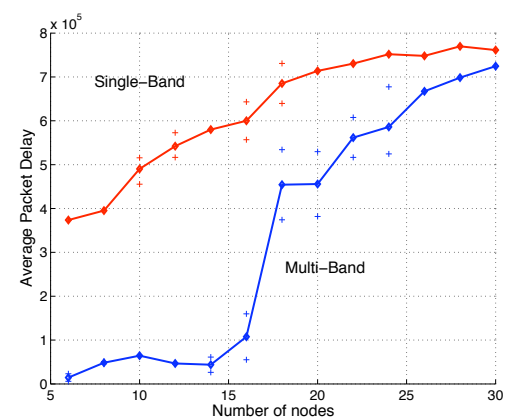

Fig. 6. Average packet delay

observations from all the nodes in the network as a function of the number of nodes. We observe a much higher (more than 4 times) bit error rate in the single-band system, again, a direct result of collisions of data packets.

Next we report the observed average packet delay in the network. The packet delay is the duration between the instance that a packet arrives to the MAC layer queue of a node, until the instance that it is completely reconstructed at its destination. This delay accounts for retransmissions that may occur due to the failure of the packet transfer due to the packet being corrupted or collided with. In figure 6 we plot the average packet delay as a function of the number of nodes in the network with CBR traffic. In our protocol, packet delays are lower by a factor of six as compared with the delay incurred with the single band scheme for low network densities (i.e., when the networks consists of 15 to 16 nodes). With more nodes, the multiband delay rapidly increases and approaches the delay that is observed with the single-band case, when there are approximately 30 nodes in the network. Note that this behavior with a large number of nodes is an artifact of the system reaching its capacity. Recall that as the number or nodes increases, the network load increases as well in this experiment.

We also measured the total number of transmitted data packets, for the duration of the simulation with CBR traffic. Note from figure 7 , that the network throughput in terms of transmitted packets is higher with the multiband scheme. Our protocol performs better by as much

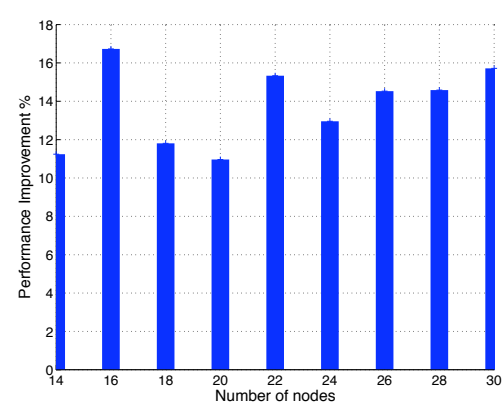

Fig. 7. Performance improvement in terms of throughput - total transmitted data packets $T$. The improvement is calculated as: Improvement $=\left(\left(T_{\text {Multiband }}-T_{\text {Singleband }}\right) / T_{\text {Singleband }}\right) * 100 \%$

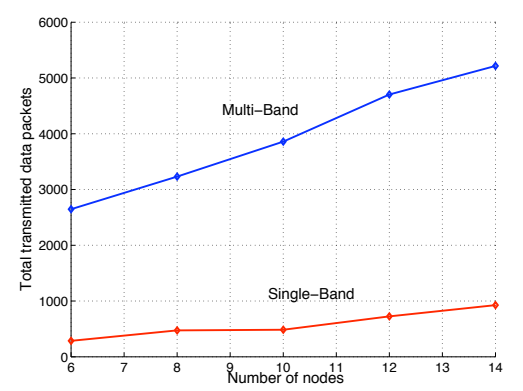

Fig. 8. Throughput improvements at heavy loads with a small number of users in the network.

as $16.72 \%$, a significant increase at these higher capacities. Furthermore notice that this improvement is over an unfairly advantaged single-band system, which magically eliminates the effects of pulse collisions on the reception of data packets and hence can support any number of simultaneous data transmissions as long as their request handshake was successful.

In all of the previous examples, we assume that the load increases with the number of users. We perform experiments to demonstrate the benefits of our scheme with high loads when the number of users in the network are small. In such cases, communicating pairs can be allocated exclusive bands in the multi-band approach. With the single band approach however, throughput is much lower due to collisions. For this experiment, we assume that packets are generated at each node at a constant rate of one every 1.4 milliseconds. We observe that, now, the achieved throughput is more than an order of magnitude better than with the single-band approach (shown in figure 8).

Our final simulation experiment examines the band occupancy with the multi-band approach. The objective of this experiment is to determine if the traffic load is uniformly distributed across the data bands or if some bands are preferentially used with our policy. The motivation for this study is that if some of the bands are hardly ever used, one might consider the usage of additional control bands to improve efficiency. For facilitating understanding, in this simulation, we assume a clique with ad hoc com- 


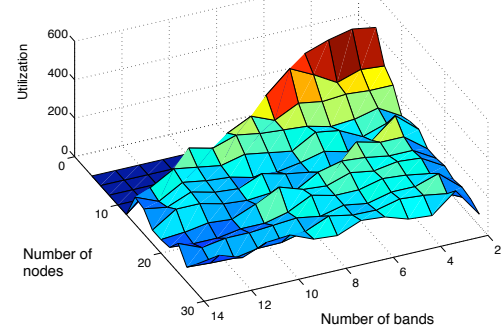

Fig. 9. Use of bands for successful transmissions, as a function of nodes

munications and we perform measurements in the cases wherein the network is very heavily loaded and the individual nodes always have packets to send. It is in this regime that we wish to observe band occupancy for the motivating reason specified above.

Initially, we assume that the band that a node chooses is determined via a simple mapping function from its identifier (ID) (a simple hash function is assumed). Later, the preferential band selection process discussed in Section III is adopted. In figure 9 we plot the number of times each band is chosen (utilization), as a function of the number of nodes in the network. When the number of nodes exceeds the number of bands, there is a tendency for all bands to be used uniformly in the long run. The highest utilization values are observed for small numbers of nodes, where no collisions in data bands occur. When all bands are used, the maximum utilization is observed for 12 nodes and decreases as the number of nodes increases. Notice that, when the number of nodes is small, with our policy, not all bands are utilized. Thus, with small scale deployments one may consider the use of more bands for dissemination of control information to improve performance. We will consider such possibilities in future work.

\section{RELATED WORK ON UWB NETWORKS}

There is no prior work on the design of a MAC protocol for multiband UWB based wireless networks that support ad hoc communications. However, there have been some interesting studies on single-band implementations.

Previous Ad Hoc UWB Schemes: Le Boudec et al. [6], [7], [8] propose a scheme that uses dynamic channel coding. The scheme uses two types of THS: a receiver-based THS and an invitation-based THS. After the successful transmission of a request using the receiver based THS, the pair switches to a unique invitation-based THS and uses this THS for the duration of the session. In [12] the authors describe theoretical and practical approaches towards the development of a THS based MAC protocol for radio resource sharing in UWB ad hoc networks. The effects of multipath and in particular delay spread are not addressed in these papers. The authors appear to implicitly assume the presence of a perfect equalizer.

Ding et al study issues related to channel acquisition [18]. They conclude that existing MAC solutions are unsuitable for UWB networks. Specifically, the authors examine TDMA and CSMA/CA approaches, which have been successful in other environments. In [27], all nodes share a THS and the receiver broadcasts an invitation, as per this sequence. Potential transmitters compete during a contention period, to lock on to the receiver. In [29] the authors propose a full-duplex access scheme for impulsebased UWB networks. The scheme takes advantage of the low duty cycle to maintain physical links among two nodes for the lifetime of their logical link, thereby removing the requirement that the sender and receiver resynchronize for every packet to be exchanged. A theoretical treatment on optimal routing, scheduling and power control appears in [9]. The authors show that (1) the design of the optimal MAC is independent of the choice of the routing protocol and (2) a minimum energy route is preferable to establishing long hops or invoking direct transmissions.

WPAN Configurations: Most other studies consider master-slave configurations [4], [28], [10]. The IEEE 805.15.3a task group proposal [4] for media access control is based on the notion of piconets. Each piconet includes a master-coordinator, which assigns resources to slaves. The task group has evaluated numerous proposals for the UWB physical layer. The Multiband OFDM Alliance (MBOA), supports a UWB specification that is based on an OFDM approach [5]. Note here that the OFDM approach attempts to reduce the equalizer complexity by dividing the available spectrum into multiple bands. However, the use of OFDM requires (i) frequent complex inverse fast fourier transform computations [14] (ii) simultaneous receiver synchronization with multiple carriers. Thus, there are trade-offs between the use of OFDM based UWB and impuse-based UWB. A MAC layer protocol for use with OFDM for ad hoc networks is yet to emerge. Yomo et al. [10] study the interference between distinct WPANs (Wireless Personal Area Networks) that operate in a master-slave configuration.

Reservation based MAC protocols: The use of reservations for arbitrating access to a plurality of orthogonal bands has been considered in wireless and satellite networks [19] [30]. However, the presence of a centralized arbiter (a satellite or base-station) makes allocation much easier as compared to allocation in ad hoc networks. Recently, the use of multiple bands in ad hoc networks that use the IEEE 802.11 MAC protocol has been considered in [31]. However, carrier sensing is possible with IEEE 802.11 and the issues related to MAC access are different 
from those that arise due to the use of UWB.

\section{CONCLUSions}

In this paper we propose a novel multiband MAC protocol for use with impulse-based UWB ad hoc networks. The design of our protocol is motivated by the following factors: (a) in the absence of a complex equalizer, due to the effects of the multipath delay spread the entire UWB spectrum cannot be efficiently utilized by a single band approach. (b) arbitration methods based on the use of time-hopped sequences suffer from inefficiencies due to collisions or large delays and, (c) the use of a multi-band approach provides an inherent flexibility in operation to coexist with other wireless networks. The approach we present is conjoint with the UWB physical layer and takes into account the regulations imposed by the FCC. We perform extensive simulations to demonstrate that our protocol achieves extremely high throughput and much lower latencies as compared to a single band approach wherein no equalizer is available.

\section{REFERENCES}

[1] Federal Communications Commission: "Revision of Part 15 of the Commission's Rules Regarding Ultra-Wideband Transmission Systems", First Report and Order, ET Docket 98-153, 04-2002.

[2] J. G. Proakis, Digital Communications, McGraw-Hill, N.Y. 2001, 4th edition.

[3] R.Gitlin, J.F.Hayes and S.Weinstein,Data Communication Principles, Plenum Press, 1992.

[4] IEEE 802.15.3 MAC standard.

[5] Multiband OFDM Physical Layer Proposal for IEEE 802.15.3a, September 2004, http://www.multibandofdm.org

[6] J.Y. Le Boudec, R. Merz, B. Radunovic and J. Widmer, "A MAC protocol for UWB Very Low Power Mobile Ad-hoc Networks based on Dynamic Channel Coding with Interference Mitigation”, EPFL Technical Report ID: IC/2004/02, 01-26-2004.

[7] J.Y. Le Boudec, R.Merz B.Radunovic and J.Widmer, 'DCCMAC: A Decentralized MAC Protocol for 802.15.4a-like UWB Mobile Ad-Hoc Networks Based on Dynamic Channel Coding" Proceedings of Broadnets, 2004.

[8] R.Merz, J.Y.Le Boudec, J.Widmer, B.Radunovic, "A RateAdaptive MAC Protocol for Low-Power Ultra-Wide Band Ad hoc Networks", Proceedings of Ad-Hoc Now, 2004.

[9] B.Radunovic and J.Y.Le Boudec 'Optimal Power Control, Scheduling and Routing in UWB Networks", IEEE Journal on Selected Areas in Communications, September 2004, Vol.22, No.7.

[10] H. Yomo, P. Popovski, C. Wijting, I.Z. Kovacs, N. Deblauwe, A.F. Baena and R. Prasad, 'Medium Access Techniques in Ultrawideband Ad Hoc Networks", 6th National Conference of Society for Electronic, Telecommunication, Automatics, and Informatics (ETAI), September 2003, Ohrid, Fyrom.

[11] ANSI/IEEE 802.11 Standard, 1999 Edition.

[12] F. Cuomo, C. Martello, A. Baiocchi and C. Fabrizio: 'Radio resource sharing for ad hoc networking with UWB" IEEE Journal on Selected Areas in Communications, December 2002.

[13] S.Roy, J.R.Foerster,V.S.Somayazulu and D.G.Leeper 'Ultrawideband Radio Design: The Promise of High-Speed, Short-Range Wireless Connectivity", Proceedings of the IEEE, February 2004.
[14] M.Ghavami, L.B. Michael and R.Kohno, Ultra Wideband Signals and Systems in Communication Engineering, John Wiley and sons, 2004.

[15] J. Foerster, E. Green, V. S. Somayazulu and D. Leeper: 'UltraWideband Technology for Short - or Medium - Range Wireless Communications", Intel Technology Journal, Q2, 2001.

[16] M. Z. Win and R.A. Scholtz: 'Impulse Radio: How It Works", IEEE Communications Letters, Vol.2, No.1, 01-1998.

[17] M. Z. Win and R. A Scholtz: 'Ultra-Wide Bandwidth TimeHopping Spread-Spectrum Impulse Radio For Wireless Multiple Access Communications", IEEE Transactions of Communications, 04-2000.

[18] J. Ding, L. Zhao, S. Medidi and K. M. Sivalingam: 'MAC Protocols for Ultra-Wide-Band Wireless Networks: Impact of Channel Acquisition Time", in SPIE-ITCOM Conference.

[19] K.Pahlavan and P.Krishnamurthy, Principles of Wireless Networks: A Unified Approach, Prentice Hall, 2002.

[20] Discrete Time Communications: 'IEEE 802.15.3a 480Mbps Wireless Personal Area Networks: Achieving a Low Complexity Multi-band Implementation", white paper, January 2003.

[21] J. J. Garcia - Luna - Aceves and J. Raju: 'Distributed Assignment of Codes for Multihop Packet - Radio Networks", IEEE MILCOM 1997, vol. 1, pp. 450-454.

[22] E. S. Sousa and J. A. Silvester: 'Spreading Code Protocols for Distributed Spread-Spectrum Packet Radio Networks", IEEE Trans. Comm., COM-36, pp 272-281, 1988.

[23] Texas Instruments et al.: 'Multi-band OFDM Physical Layer Proposal for IEEE 802.15 Task Group 3a”, Sept. 2003.

[24] M. E. Crovella and A. Bestavros. "Self-Similarity in World Wide Web Traffic: Evidence and Possible Causes". In IEEE/ACM Transactions on Networking, 1997.

[25] K.Romer. "Time Synchronization in Ad Hoc Networks". In ACM MOBIHOC 2001.

[26] L. Meier, P.Blum and L.Thiele. 'Internal Synchronization of Drift-Consistent Clocks in Ad-Hoc Sensor Networks", In ACM Mobihoc 2004.

[27] A. Hicham, Y. Souilmi and C. Bonnet: 'Self-balanced receiveroriented MAC for ultra-wide band mobile ad hoc networks", International Workshop on Ultra Wideband Systems, June 2003.

[28] F. Cuomo, A. Baiocchi, F. Capriotti, C. Martello, 'Radio Resource Optimisation in an UWB Wireless Access", Proceedings of IST Mobile Communications Summit 2002, Thessaloniki, Greece, June 17-19 2002, pp. 723-727.

[29] S. Kolenchery, J. Townsend and J. Freebersyser, "A Novel Impulse Radio Network for Tactical Military Wireless Communications", in IEEE MilCom 1998.

[30] S.Krishnamurthy, C.Liu and V. Gupta, "Medium Access Control Protocols for Satellite Communications", in Internetworking and Computing over Satellite Networks, Y.Zhang ed. Kluwer, 2003.

[31] J.So and N.Vaidya, "Multi-Channel MAC for AdHoc Networks: Handling Multi-Channel Hidden Terminals Using A Single Transceiver", ACM MOBIHOC, 2004. 